\title{
Primate Memory
}

\author{
Tetsuro Matsuzawa
}

My life as a primatologist and researcher has been spent studying chimpanzees, both in the wild and in the laboratory. Our closest living relatives in the animal world are fascinating creatures. In this essay, I recount some of the most striking aspects of my work to illustrate the development of my views about primates, memory, and the evolution of the human mind.

$\mathrm{T}$ HE MOST RECENT common ancestor of chimpanzees and humans lived between five and seven million years ago. This shared heritage became evident when sequencing revealed a 1.2\% DNA difference between species. ${ }^{1}$ Chimpanzees have a living sister species, bonobos, that is equally closely related to humans. ${ }^{2}$ Both chimpanzees and bonobos are found only in Africa; this is also true of gorillas. Chimpanzees and humans shared a common ancestor with gorillas between eight and nine million years ago. Another species of great ape closely related to humans are orangutans, which live in Southeast Asia, mostly on the islands of Borneo and Sumatra.

The family Hominidae consists of four genera: humans, chimpanzees (and bonobos), gorillas, and orangutans. The most recent common ancestors of hominids is thought to have lived around fourteen million years ago. In 2019, the International Union for Conservation of Nature listed no less than 512 living species of primates belonging to seventy-nine genera. These species were previously classified as hominids, gibbons, old-world (Afro-Eurasian) monkeys, new-world (American) monkeys, lemurs, and prosimians. With the exception of humans, all the living species of primates reside in Africa, Asia, and Central and South America.

Japan has its own indigenous monkey species in the Japanese macaque, which are sometimes referred to as snow monkeys. The monkeys found in the Shiga Kogen area of central Japan have become famous as the only species known to bathe in hot springs during winter. Another monkey community living on the tiny island of Kōjima near the southern tip of Japan is known for washing sweet potatoes. To wash away the sand from the potatoes they pick up from the beach, the monkeys carry the potatoes to the shallows and dip them in the sea, where they acquire a salty taste. This behavior was first observed in 1953 and is passed from one generation of monkeys to the next. The sweet-potato washing observed on Kōjima was the first example of cultural behavior found in nonhuman animals. ${ }^{3}$

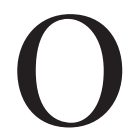
VER THE COURSE of my career, I have undertaken numerous studies investigating the use of tools by chimpanzees in the wild. In recent years, this research has centered on a community of chimpanzees living in the woodlands around the town of Bossou in the West African country of Guinea. These chimpanzees have developed a unique method of gathering drinking water from tree hollows. The technique involves using their mouths to fold leaves and create a receptacle for the water. ${ }^{4}$ The appearance of the folded leaves has been compared to origami. There are numerous other examples of tool use within the group. The same community use a pair of stones as a hammer and an anvil to crack open oil palm nuts and gain access to the edible kernel. ${ }^{5}$ I tried the kernel for myself and found it to be quite tasty. Another example involves the chimpanzees using a twig as a fishing stick to gather safari ants from both the ground and their nests. I also tried eating safari ants and found that they are crunchy but may bite your tongue.

The Bossou chimpanzees have also found a novel approach for scooping up algae floating on the surface of a pond. Tatyana Humle and I discovered this behavior in 1995. ${ }^{6}$ After following a group of chimpanzees to a glade of tall grass, we observed a four-year-old female that we had named Fotaiu stepping into the thick vegetation surrounding a pond. She then began manufacturing a scooping tool. The first step was choosing a fern (Cyclosorus afer, Polypodiaceae) from among the plants growing in the glade and finding a stalk on the ground. Fotaiu then bit off the distal end of the stalk, using her teeth to shorten it to around fifty centimeters. While holding the stalk in her mouth, she stripped off the leaves along its stem using one hand in a swift, downward motion. ${ }^{7}$ Holding the stick between her index and middle fingers, she dipped it into the pond and made a swiveling action with her wrist, moving the stick back and forth to collect the floating algae. She then lifted the stick to her mouth and pulled it sideways through her lips, gathering the algae in her mouth. ${ }^{8} \mathrm{I}$ have attempted to 
mimic this approach with mixed results. I also tasted the algae and found it muddy and rather unpalatable.

In 1960, Jane Goodall was the first to observe wild chimpanzees engaged in termite fishing. ${ }^{9}$ During the six decades since that first sighting in Gombe, Tanzania, many further examples of tool manufacture and use among chimpanzees have been discovered. It has become clear that each chimpanzee community has its own repertoire of tools and associated behaviors. ${ }^{10}$ The Bossou chimpanzees pick up and eat termites as they emerge from their mounds, but have not been observed using tools to extract termites hidden inside these structures. Even though stones, nuts, and algae are all readily available for the chimpanzees in Gombe, there is no evidence that they use stone tools to crack open oil-palm nuts, nor have they been observed engaging in algae scooping.

$\mathrm{I}$ N MY OWN WORK, I have often described the social learning techniques of chimpanzees as education by master-apprenticeship. ${ }^{11}$ Mothers and other adults take on the role of the master. The young chimpanzees in the community learn by carefully observing the behavior of the masters. Observational learning has three important aspects: the master models behavior but does not actively teach it; the apprentice has a strong and intrinsic motivation to copy the behavior; and, importantly, the masters are tolerant toward their apprentices while they learn.

Consider the learning process involved in stone tool use. Despite their best efforts, the first attempts made by infant chimpanzees to crack nuts end in failure. It is not until they are around four or five years of age that young chimpanzees are able to start cracking nuts for themselves. To compensate for this shortcoming, very young chimpanzees are allowed to steal kernels from their mothers. I have observed young infants stealing up to seven kernels from the mother in quick succession. As the infants grow, they steal fewer and fewer kernels. At the same time, their attempts at using stones to crack nuts become more frequent and varied. This demonstrates that the motivation for young chimpanzees to adopt the same behaviors as their mothers and other adults is not solely derived from the need for sustenance. ${ }^{12}$

The Bossou forests are home to around 600 plant species. About a third of these are used by chimpanzees, who eat their fruits and leaves, as well as their flowers, stems, roots, and bark. Confronted with so many different plant species, it may take a long time for young chimpanzees to acquire knowledge about plants and the skills that they need to survive in the forest. ${ }^{13}$ As part of my fieldwork in Bossou, I tracked chimpanzees between 6:30 in the morning and 6:30 in the evening. With the help of local assistants, I was able to learn about their daily activities and social life. ${ }^{14}$ I was often amazed to discover how well the chimpanzees knew when particular fruits were available and where to find them.
$\mathrm{F}$ IELD STUDIES ARE helpful to learn more about the cognition and behavior of chimpanzees. But they are less well suited for more detailed investigations of their cognitive functions. How do chimpanzees distinguish red fruits from the surrounding green leaves? How do they memorize their experiences in the forest? Are their cognitive processes similar to those of humans, or completely different? These questions are all best examined in a laboratory setting.

The nineteenth-century physicist and philosopher Gustav Fechner is considered one of the founders of experimental psychology. Fechner was the leading figure in the development of psychophysics, a discipline focused on the relationship between perceptual and physical worlds. The Weber-Fechner laws are named after the physician, Ernst Weber, and his student, Fechner. The perceived heaviness of an object, for example, is not simply proportional to its weight, nor is the relationship linear. Instead, Fechner found that the relationship between the perceived weight and the actual weight is a logarithmic function.

The nature of memory also emerged as one of the central issues in early psychological studies. Hermann Ebbinghaus was the first to apply psychophysics to its experimental study. He invented a memory test using three-letter nonsense syllables such as SIF, PIJ, RIT, and TAS. To find out how much time was needed to memorize these syllables, Ebbinghaus became his own test subject. By analyzing the results of these tests, he was able to discern the forgetting curve plotting memory decay over time, and identify factors that helped account for shrinking retention.

The knowledge and skills acquired by chimpanzees in their natural habitats are retained in long-term memory. In humans, a distinction is made between two categories of long-term memory: declarative (explicit) memory and procedural (implicit) memory. Declarative memory stores factual and episodic information. Procedural memory makes it possible to recall how to do things, such as swimming. ${ }^{15}$ From my own observations in the field, I am persuaded that chimpanzees in the wild possess both categories of long-term memory. A more rigorous experimental approach is needed to determine precisely what kinds of memories are retained by chimpanzees, and how similar or different those might be to the memories of humans. ${ }^{16}$

In parallel with my fieldwork and research, I have long been involved in laboratory testing using involving captive chimpanzees. ${ }^{17}$ The Primate Research Institute of Kyoto University (KUPRI) has hosted a long-running study aimed at elucidating the cognitive capacities and intelligence of chimpanzees. The main test subject is a female chimpanzee, Ai.

Ai was born in Africa in 1976. When she arrived at KUPRI, she was approximately one year old. ${ }^{18}$ I first met the tiny chimpanzee in the basement of the institute in November 1977. The Ai project began under the leadership 
of my mentor, Kiyoko Murofushi, in collaboration with Toshio Asano and myself. As the youngest professor on the team, I assumed responsibility for the daily care and testing of chimpanzees. A key feature of the Ai project that has remained constant is a simplified experimental setting involving a chimpanzee seated in front of an automated device, with little or no direct human involvement. ${ }^{19}$

Although the Ai project was originally conceived as an ape-language study, I hoped it might be possible to use the same language system to study the perceptual world of chimpanzees. My goal was to compare the perceptual worlds of chimpanzees and humans using the same methods and following the same procedures. By establishing chimpanzee psychophysics as a discipline in its own right, I sought to create the basis for a broader study in comparative cognitive science examining the minds of humans and nonhuman animals. ${ }^{20}$

$\mathrm{W}$

E BEGAN THE project with three young chimpanzees-Ai, Akira, and Mari-when they were between 1.5 and 2.5 years of age..$^{21}$ During the experiments, the chimpanzee sat in a test booth with a backlit keyboard configured in a $5 \times 7$ matrix. Each key was about the size of a fingertip. The keys were illuminated when they were available and kept dark when they were inactive. On April 15, 1978, Ai pressed a key for the first time.

To begin with, the chimpanzees needed to adapt to both the test booth and the keyboard. The first task was to press an illuminated key. The chimpanzees were then prompted to perform an identity matching-to-sample task. When a sample color-say, red-appeared on the small screen of the projector, the chimpanzees had to press the corresponding red-colored key on the keyboard. The chimpanzees subsequently learned to discriminate between three colors: red, green, and blue. They also learned to discriminate between nine elemental figures, or graphemes: $(\mathrm{H})$ orizontal line, (O)blique line, (W)ave, (S)nake, (B)lock, (D)ot, (L) ozenge, (C)ircle, and (R)ectangle.

As part of the project, I came up with the Kyoto University Lexigram system (KUL). By combining two or three graphemes, it becomes possible to create a unique visual pattern that can be used as a symbol. The idea behind KUL comes from the Japanese-Chinese letters used in kanji. Each kanji letter can be broken down into elements known as radicals. An object such as "pencil" is represented in KUL by combining the graphemes for B and R. The color blue, for example, is represented by DC, while black is OLR.

The chimpanzees also learned a symbolic matching-to-sample task. The sample was the real object, shown to them by the experimenter. The objective was to press the key with the corresponding lexigram. Ai required fifty-seven daily sessions to learn the symbols for eight objects: ball, brick, bowl, glass, glove, paper, pencil, and toothbrush. Akira took eighty-three days, and Mari took 104 days. They also learned the reciprocal use of the lexigrams, decoding the lexigrams to match to the real objects. Both the productive use and receptive use of the lexigrams may provide the basis for symbolic thinking.

At the age of four, Ai expanded her repertoire of learned symbols to include eleven color names: red, orange, yellow, brown, green, blue, purple, pink, white, grey, and black. ${ }^{22}$ Each color name was represented by a lexigram. Later in the project, when touch screens became available, the lexigrams were replaced with the kanji characters representing the eleven colors. ${ }^{23}$ Each color name was taught using a specific sample color. I tested the categorization of the various colors using 224 pieces of Munsell color chips that differed in hue, saturation, and brightness. Working in the 1960s, Brent Berlin and Paul Kay found that the universality of color terms outweighed cultural differences. ${ }^{24}$ Ai's results showed that the universality of color categorization is a trait shared by both species. Chimpanzees memorize color terms just like humans.

\section{Figure 1.}

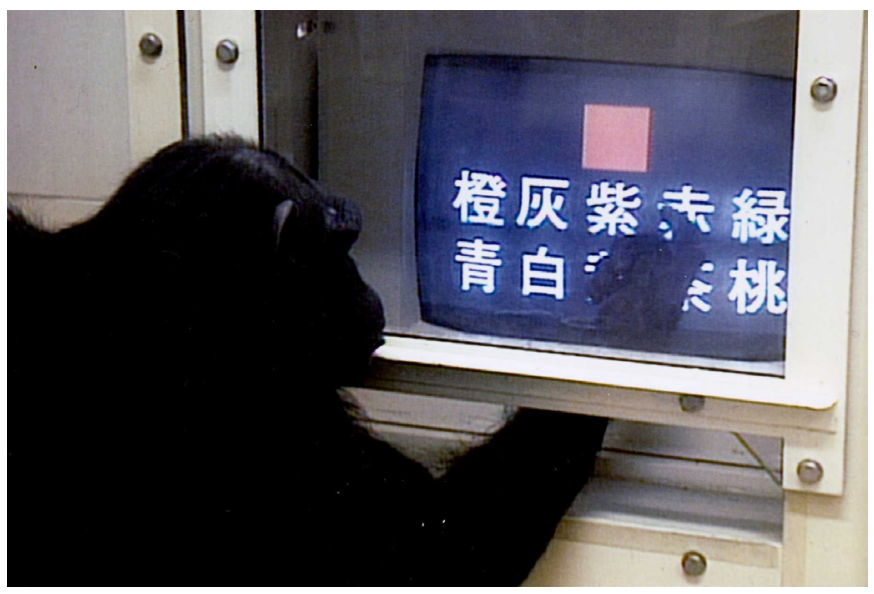

A sample of the color red is displayed for Ai on the touchscreen. She is about to touch the corresponding kanji letter for "red." Photo by the author.

At the age of five, Ai learned that Arabic numerals can represent numbers. ${ }^{25}$ She was the first chimpanzee to successfully label numbers. Combining her acquired skills of object and color naming, Ai can assign the label "Red/ Pencil/5" when five red pencils are shown to her. Her spontaneous word order preference was either colorobject-number or object-color-number; the number was always placed at the last position in the three-word naming schema.

At the age of six and a half, Ai started learning the letters of the alphabet. After some time, she was able to discriminate between all twenty-six uppercase letters. ${ }^{26}$ Each of the letters was available on a keyboard, meaning that the chimpanzee keyboard was essentially the same as a reg- 
ular computer keyboard. In the initial phase of learning, she confused letters such as E and F, D and O, O and Q, V and $\mathrm{Y}$, and $\mathrm{M}$ and $\mathrm{W}$. It seems that these shape pairs have a similar appearance for chimpanzees. Once learned, the letters were also used to measure visual acuity. Placed at a distance from the chimpanzee, the letters were gradually reduced in size to determine the threshold of detection. The visual acuity of Ai was found to be 1.5, which is comparable to that of humans with normal eyesight. In a subsequent test, the letters represented the names of individual humans and chimpanzees. The letter $\mathrm{A}$, for example, was allocated to chimpanzee Akira, $M$ was for Mari, and $\mathrm{Z}$ was for Matsuzawa. For Ai, recognizing the faces of chimpanzees was easier than those of humans, while the human subjects showed greater difficulty in identifying chimpanzee faces. ${ }^{27}$

At the age of seven and a half, Ai learned the lexigrams for apple (consisting of the graphemes DCR), banana (SLC), carrot (HBC), cabbage (BDL), potato (OSB), and chow (HWS) as part of a symbolic matching-to-sample task. She also learned symbols for six food items. She then completed a constructive matching-to-sample task in which the sample was a lexigram and Ai had to match the sample using the nine graphemes on the keyboard. Finally, she was tested using another task known as symbolic constructive matching-to-sample. When a real apple was shown to Ai as a sample, she had to construct the corresponding lexigram by choosing the graphemes D, C, and $\mathrm{R}$ on the keyboard. On the very first trial of the first day of testing, she constructed the lexigram for carrot from the nine graphemes. On average, she needed 2.8 attempts to construct the corresponding lexigrams for six food items upon first encounter. ${ }^{28}$ The Ai project proved that the chimpanzees have sufficient long-term memory to learn double-segmented symbol systems-making a symbol from scratch, such as food names from graphemes, and combining the symbols into a noun phrase, such as red/ pencils $/ 5$.

The Ai project developed into an integrated research effort that continues to the present day. Current research efforts are focused on a multigenerational group of twelve chimpanzees. The forty-five-year history of the Ai project has now included several generations of scientists, chimpanzee participants, research topics, and technological methods..$^{29}$ It has also emphasized the importance of maintaining a naturalistic physical and social environment for the chimpanzee subjects, including the development of a seminatural living space with fifteen-meter-high climbing towers in an outdoor compound, connections between multiple habitats, and a multigenerational social group of conspecifics. ${ }^{30}$

$\mathrm{I}$

N 2000, AT THE age of twenty-three, Ai gave birth to a son, Ayumu. Ayumu's father was Akira, a long-term friend of Ai. Two other females in the group also gave birth that year. As a result, we were able to study three mother-infant pairs at the same time. Since then our research has been mainly concerned with cognitive development. ${ }^{31}$ Studies investigating memory have been the most important aspect of our work involving young chimpanzees. It has been short-term memory, or working memory, rather than long-term memory that has been our main focus.

In tests where three numerals-say, 3, 5, and 8-were presented on a touch screen, all the chimpanzees tested were able to touch the numerals in ascending order. We were amazed by the speed at which they completed the task. A postdoctoral student, Dora Biro, now an Oxford University professor, came up with an interesting short-term memory test that involved switching the second and third numerals immediately after the first was touched. ${ }^{32}$ When 3 was touched, for example, the positions of 5 and 8 were swapped. In these tests, the chimpanzees often made the error of touching 3 and then touching 8 instead of 5 . The results demonstrated that the chimpanzees had memorized the three numerals at a glance and had planned the order to touch them in advance.

The ability to memorize three numerals at a glance led to another question: How many numerals can chimpanzees memorize in this manner? Human cognition includes a process known as subitizing. For up to five items, we do not need to count them one by one; we can immediately recognize the number of items at a glance. George Miller, one of the founders of cognitive science, was the first to point out human short-term memory was, on average, limited to seven items. ${ }^{33}$ A postdoctoral student working on the project, Nobuyuki Kawai, now a professor at Nagoya University, proposed a new test that we named the masking task. ${ }^{34}$ Kawai's test was a modified version of the swapping trial: when the first numeral was touched, the second and third numerals were immediately masked by white rectangles. When 3 was touched, for example, 5 and 8 were replaced with white rectangles. The goal was for the chimpanzees to touch the white rectangle where 5 had been. The masking task with three numerals can be easily extended to a masking task with four numerals-using a sequence such as 3, 5, 8, 9-and a masking task with five numerals, and so on. Ai's performance on masking tasks involving four and five numerals was comparable to that of human adults.

While conducting these tests, we were amazed by the performance of the three young chimpanzees: Ayumu, Cleo, and Pal, all of whom were four years of age. Their performance on the masking task was much better than that of their mothers. Ayumu was even able to complete a masking task involving nine numerals. ${ }^{35}$ Humans are typically able to complete this task if they spend ten to twenty seconds memorizing the positions of each numeral from 1 to 9.36 
We then decided to make a direct comparison between the working memory of humans, adult chimpanzees, and young chimpanzees. The masking task was modified slightly for the purposes of this comparison. Sana Inoue, now an associate professor of Ritsumeikan University, and a postdoctoral student at that time, introduced the limited-hold task, in which the numerals were presented to the chimpanzees for only a brief duration. ${ }^{37}$ Suppose that there are five numerals-2, 3, 5, 8, and 9-displayed on the touch screen. After 650 milliseconds (ms), $430 \mathrm{~ms}$, or $210 \mathrm{~ms}$, all the numerals are automatically replaced by white squares. The goal is to touch the white squares in the ascending order of the now-masked numerals.

\section{Figure 2.}
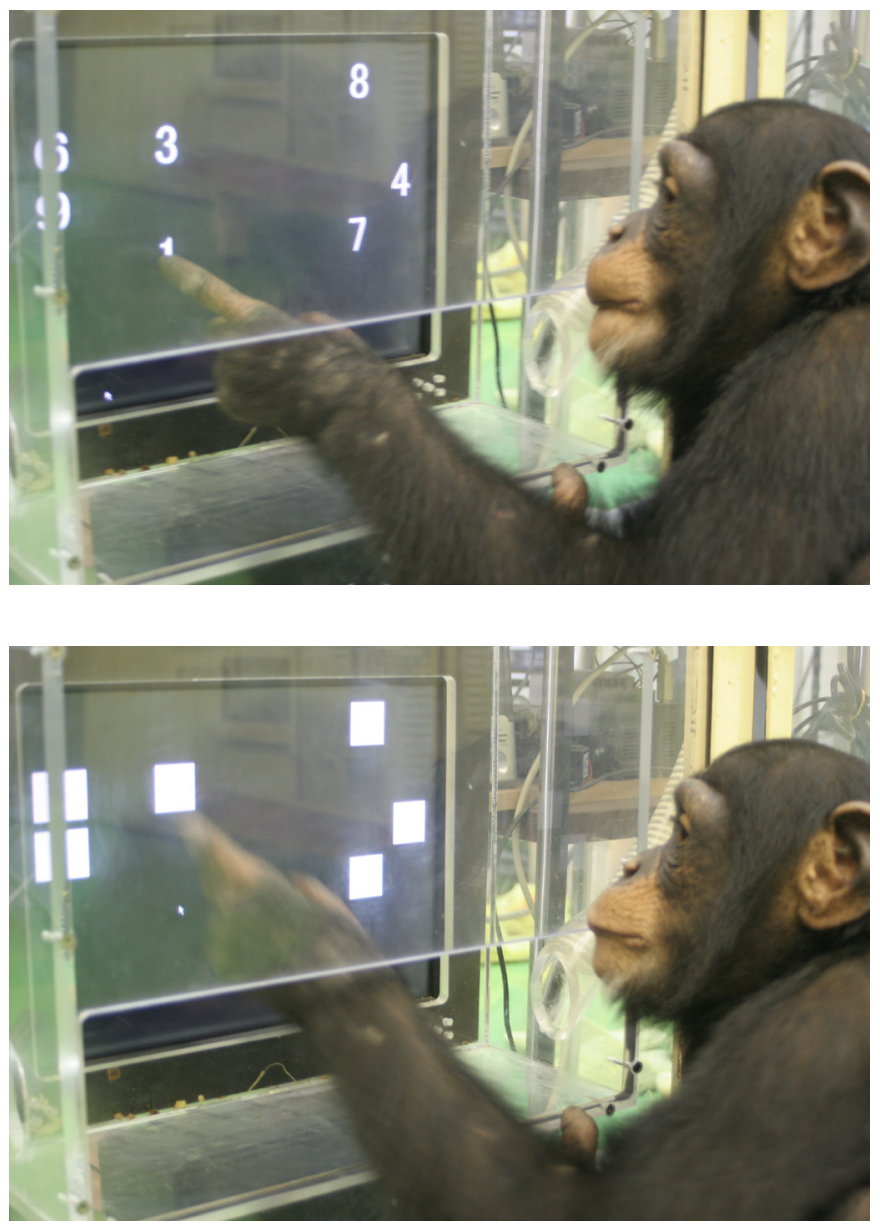

Ayumu is shown working on the masking task involving seven numerals. As soon as Ayumu touches the first numeral, all the rest are replaced with white squares. Photos by the author.

Ai's performance in the limited-hold task was comparable to that of university students facing the same test for the first time. The performances of three young chimpanzees were much better than those of humans. We also tested the impact of overtraining among human subjects, allowing them to repeat the memory test many times over.
Although their performances improved with practice, no human has ever been able to match Ayumu's speed and accuracy in touching the nine numerals in the masking task. $^{38}$

One day, a chance event occurred that illustrated the retention of working memory in chimpanzees. While Ayumu was undertaking the limited-hold task for five numerals, a sudden noise occurred outside. Ayumu's attention switched to the distraction and he lost concentration. After ten seconds, he turned his attention back to the touch screen, by which time the five numerals had already been replaced with white squares. The lapse in concentration made no difference. Ayumu was still able to touch the squares in the right order. This incident clearly shows that the chimpanzee can memorize the numerals at a glance, and that their working memory persists for at least ten seconds.

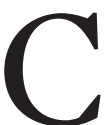

HIMPANZEES ARE ABLE to remember the meaning of visual symbols, such as lexigrams, kanji, letters of the alphabet, and Arabic numerals. Despite this capability, the proto-linguistic capability of chimpanzees, although it clearly exists, remains limited. There have been a number of attempts to teach chimpanzees human methods of communication, such as vocal communication, gestural sign languages, plastic sign languages, lexigrams, and so on. ${ }^{39}$ In these studies, chimpanzees have been unable to reach the same level of proficiency as humans. The Ai project proved that although chimpanzees were not good at memorizing arbitrary associations of symbols and their corresponding meanings, they were very good at memorizing items at a glance.

Recent research using eye-tracking technology has revealed some interesting differences between humans and chimpanzees. As part of these studies, no training or verbal instruction was provided other than requesting that the participants remain seated in front of a monitor. A small visual stimulus, such as a face, is displayed in a random position on the screen and is quickly changed in succession to other images in different positions. The participants observe and spontaneously track the ever-changing stimuli. The studies have shown that chimpanzees possess a remarkable aptitude for switching focus from one stimulus to another. This is not the case for humans. We often stick to one stimulus and are unable to follow the quick change to the next. It seems that our way of perceiving the world is highly skewed toward focusing on a stimulus to determine its meaning. By contrast, chimpanzees are able to shift their attention to capture the whole scene as quickly as possible. ${ }^{40}$

In 2013, I proposed the cognitive tradeoff theory of language and memory. ${ }^{41}$ Our most recent common ancestor with chimpanzees may have possessed an extraordinary chimpanzee-like working memory, but over the course of human evolution, I suggested, we have lost this capability 
and acquired language in return. ${ }^{42}$ Suppose that a creature passes in front of you in the forest. It has a brown back, black legs, and a white spot on its forehead. Chimpanzees are highly adept at quickly detecting and memorizing these features. Humans lack this capability, but we have evolved other ways to label what we have witnessed, such as mimicking the body posture and shape of the creature, mimicking the sounds it made, or vocally labeling it as, say, an antelope.

Our human ancestors are thought to have moved out of the forests and onto the savannah millions of years ago. ${ }^{43}$ In doing so, they developed a collaborative way of living characterized by group hunting and male-female division of labor for raising offspring. These arrangements are quite different for chimpanzees, among whom only the mothers raise the offspring. Very little in the way of parental care is provided by the father, the grandparents, or other potential helpers. Female chimpanzees start their pregnancies at around twelve years, with an inter-birth interval of about five years. The lifespan of female chimpanzees can be fifty years or more, but there is no social role for grandmothers in their communities. ${ }^{44}$ Humans need to share information for survival; transmission of knowledge, skills, and experience from one generation to the next was essential. Although humans may have lost the power of immediate memory we see in chimpanzees, we have acquired greater powers of imagination. ${ }^{45}$ These capabilities provide the fundamental basis for language and the ability to discern, to varying degrees, the thoughts of others. In my view, it is the intrinsic motivation for sharing based on the power of imagination that is the evolutionary driving force that has made us human.

Tetsuro Matsuzawa is a Visitor in Psychology at the California Institute of Technology and former Director of the Primate Research Institute of Kyoto University.

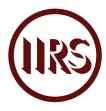

1. The Chimpanzee Sequencing and Analysis Consortium, "Initial Sequence of the Chimpanzee Genome and Comparison with the Human Genome," Nature 437 (2005): 69-87, doi:10.1038/nature04072; and Kay Prüfer et al., "The Bonobo Genome Compared with the Chimpanzee and Human Genomes," Nature 486 (2012): 527-31, doi:10.1038/ naturel1128.

2. Takayoshi Kano, The Last Ape: Pygmy Chimpanzee Behavior and Ecology (Redwood City: Stanford University Press, 1992); Christophe Boesch, Gottfried Hohmann, and Linda Marchant, Behavioural Diversity in Chimpanzees and Bonobos (Cambridge: Cambridge University Press, 2002); Brian Hare and Shinya Yamamoto, Bonobos: Unique in Mind, Brain, and Behavior (Oxford: Oxford University Press, 2017); and Takeshi Furuichi, Bonobo and Chimpanzee (Tokyo: Springer, 2019).

3. For a history of primatology in Japan, see Tetsuro Matsuzawa and William McGrew, "Kinji Imanishi and 60 Years of Japanese Primatology," Current Biology 18, no. 14 (2008): R587-91, doi:10.1016/j.cub.2008.05.040; Tetsuro Matsuzawa and Juichi Yamagiwa, "Primatology: The Beginning," Primates 59 (2018): 313-26, doi:10.1007/s10329-018-0672-9. On the topic of hot-spring bathing, see Tetsuro Matsuzawa, "Hot-Spring Bathing of Wild Monkeys in Shiga-Heights: Origin and Propagation of a Cultural Behavior," Primates 59 (2018):209-13, doi:10.1007/s10329-018-0661-z. On sweet-potato washing, see Satoshi Hirata, Kunio Watanabe, and Masao Kawai, “'Sweet-Potato Washing' Revisited," in Primate Origins of Human Cognition and Behavior, ed. Tetsuro Matsuzawa (Tokyo: Springer, 2001), doi:10.1007/978-4-43109423-4_24.

4. Cláudia Sousa, Dora Biro, and Tetsuro Matsuzawa, "LeafTool Use for Drinking Water by Wild Chimpanzees (Pan troglodytes): Acquisition Patterns and Handedness," Animal Cognition 12 (2009): 115-25, doi:10.1007/s10071-009-0278-0.

5. Tetsuro Matsuzawa, "Field Experiments on Use of Stone Tools by Chimpanzees in the Wild," in Chimpanzee Cultures, ed. Richard Wrangham et al. (Cambridge: Harvard University Press, 1994), 351-70; Noriko Inoue-Nakamura and Tetsuro Matsuzawa, "Development of Stone Tool Use by Wild Chimpanzees (Pan troglodytes)," Journal of Comparative Psychology 111, no. 2 (1997): 159-73, doi:10.1037/0735-7036.111.2.159; Dora Biro et al., "Cultural Innovation and Transmission of Tool Use in Wild Chimpanzees: Evidence from Field Experiments," Animal Cognition 6 (2003): 213-23, doi:10.1007/s10071-003-0183-x; and Susana Carvalho et al., "Chaînes Opératoires and Resource-Exploitation Strategies in Chimpanzee (Pan troglodytes) Nut Cracking," Journal of Human Evolution 55 (2008): 148-63, doi:10.1016/j.jhevol.2008.02.005.

6. Humle was an undergraduate student at Edinburgh University at that time. She is now a Reader in Conservation and Primate Behaviour at the University of Kent and the International Primatological Society's Vice President for Conservation.

7. Tetsuro Matsuzawa, "Chimpanzees Foraging on Aquatic Foods: Algae Scooping in Bossou," Primates 60 (2019): 31719, doi:10.1007/s10329-019-00733-0.

8. For footage of this algae-scooping behavior, see "Algae Scooping," YouTube video, May 31, 2012.

9. Jane Van Lawick-Goodall, "The Behaviour of Free-Living Chimpanzees in the Gombe Stream Reserve," Animal Behaviour Monographs 1, no. 3 (1968): 161-311, doi:10.1016/ S0066-1856(68)80003-2; and Jane Goodall, The Chimpanzees of Gombe: Patterns of Behavior (Cambridge: Belknap Press, 1986).

10. William McGrew, Chimpanzee Material Culture:Implications for Human Evolution (Cambridge: Cambridge University Press, 1992); and Andrew Whiten et al., "Cultures in Chim- 
panzees," Nature 399 (1999): 682-85, doi:10.1038/21415.

11. Tetsuro Matsuzawa et al., "Emergence of Culture in Wild Chimpanzees: Education by Master-Apprenticeship," in Primate Origins of Human Cognition and Behavior, ed. Tetsuro Matsuzawa (Tokyo: Springer, 2001), doi:10.1007/978-4-43109423-4_28. The idea of education by master-apprenticeship was also introduced by Frans de Waal in his The Ape and the Sushi Master: Cultural Reflections by a Primatologist (New York: Basic Books, 2001).

12. For more about the learning processes involved in stone tool use, see "Education by Master-Apprenticeship," YouTube video, March 23, 2012.

13. Yukimaru Sugiyama and Jeremy Koman, “A Preliminary List of Chimpanzees' Alimentation at Bossou, Guinea," Primates 28 (1987): 133-47, doi:10.1007/BF02382192; and Yukimaru Sugiyama and Jeremy Koman, "The Flora of Bossou: Its Utilization by Chimpanzees and Humans," African Study Monograph 13, no. 3 (1992): 127-69, doi:10.14989/68093.

14. Tetsuro Matsuzawa, Tatyana Humle, and Yukimaru Sugiyama, The Chimpanzees of Bossou and Nimba (Tokyo: Springer, 2011).

15. Endel Tulving, "Episodic and Semantic Memory," in Organization of Memory, ed. Endel Tulving and Wayne Donaldson (New York: Academic Press, 1972), 381-403.

16. Kazuo Fujita and Tetsuro Matsuzawa, "Delayed Figure Reconstruction by a Chimpanzee (Pan troglodytes) and Humans (Homo sapiens)," Journal of Comparative Psychology 104, no. 4 (1990): 345-51, doi:10.1037/0735-7036.104. 4.345 .

17. Tetsuro Matsuzawa, “The Ai Project: Historical and Ecological Contexts," Animal Cognition 6 (2003): 199-211, doi:1007/ s10071-003-0199-2; and Tetsuro Matsuzawa, "The Chimpanzee Mind: In Search of the Evolutionary Roots of the Human Mind," Animal Cognition 12 (2009): 1-9, doi:10.1007/ s10071-009-0277-1.

18. These events occurred prior to 1980, when Japan ratified the Convention on International Trade in Endangered Species of Wild Fauna and Flora.

19. Prior to the Ai project, we used a DEC-PDP-8 minicomputer to test learning capabilities among monkeys. Asano created the operating system, and I learned the machine code to operate it. The commands were similar to the nonsense syllables created by Ebbinghaus, such as TAD, DCA, ISZ, and JMS. To run the cognitive tests, we needed to switch on lights for different keys and record the key presses by the chimpanzee. The interface itself was built from transistors, resistors, condensers, and electromagnetic relays-integrated circuits were still gaining popularity at the time. The Ai project started out using a PDP-11/V03 minicomputer connected to a keyboard. At the time, floppy disks were replacing paper tape readers for data input.

20. Tetsuro Matsuzawa, The Perceptual World of a Chimpanzee (Tokyo: University of Tokyo Press, 1991) (in Japanese).

21. Toshio Asano et al., "Object and Color Naming in Chimpanzees," Proceedings of the Japan Academy, Series B 58, no. 5
(1982): 118-22, doi:10.2183/pjab.58.118; and Tetsuya Kojima, "Generalization Between Productive Use and Receptive Discrimination of Names in an Artificial Visual Language by a Chimpanzee," International Journal of Primatology 5 (1984): 161-82.

22. Tetsuro Matsuzawa, "Colour Naming and Classification in a Chimpanzee (Pan troglodytes)," Journal of Human Evolution 14, no. 3 (1985): 283-91, doi:10.1016/S0047-2484(85)80069-5.

23. Toyomi Matsuno, Nobuyuki Kawai, and Tetsuro Matsuzawa, "Color Classification by Chimpanzees (Pan troglodytes) in a Matching-to-Sample Task," Behavioural Brain Research 148, no. 1-2 (2004): 157-65, doi:10.1016/S0166-4328(03)00185-2; and Camille Pene, Akiho Muramatsu, and Tetsuro Matsuzawa, "Color Discrimination and Color Preferences in Chimpanzees (Pan troglodytes)," Primates 61 (2020): 40313, doi:10.1007/s10329-020-00790-w.

24. In their book Basic Color Terms, Berlin and Kay proposed an evolutionary account for color terms. Brent Berlin and Paul Kay, Basic Color Terms: Their Universality and Evolution (Berkeley: University of California Press, 1969).

25. Tetsuro Matsuzawa, "Use of Numbers by a Chimpanzee," Nature 315 (1985): 57-59, doi:10.1038/315057a0.

26. Tetsuro Matsuzawa, "Form Perception and Visual Acuity in a Chimpanzee," Folia Primatologica 55 (1990): 24-32, doi:10.1159/000156494.

27. This is referred to as the other-race effect in human subjects. There is another phenomenon termed the face-inversion effect. Faces presented upside down are harder to recognize than those presented right-side up. Ai showed a reduction or loss of such difficulty in naming inverted faces. Chimpanzees use arboreal habitats, in which they often hang upside down. In such an environment, the recognition of inverted faces may serve an important biological function. See Masaki Tomonaga, Shoji Itakura, and Tetsuro Matsuzawa, "Superiority of Conspecific Faces and Reduced Inversion Effect in Face Perception by a Chimpanzee," Folia Primatologica 61 (1993): 110-14, doi:10.1159/000156737. The face-inversion effect in humans and nonhuman primates remains controversial. See Christoph Dahl et al., “The Face Inversion Effect in Non-Human Primates Revisited - An Investigation in Chimpanzees (Pan troglodytes)," Scientific Reports 3 (2013): 2,504, doi:10.1038/srep02504.

28. Tetsuro Matsuzawa, "Spontaneous Pattern Construction in a Chimpanzee," in Understanding Chimpanzees, ed. Paul Heltne and Linda Marquardt (Cambridge: Harvard University Press, 1989), 252-65.

29. Chris Martin and Ikuma Adachi, "Automated Methods and the Technological Context of Chimpanzee Research," in Chimpanzee in Contexts: A Comparative Perspective on Chimpanzee Behavior, Cognition, Conservation, and Welfare, ed. Lydia Hopper and Stephen Ross (Chicago: The University of Chicago Press, 2020), 182-207.

30. Tetsuro Matsuzawa, "WISH Cages: Constructing Multiple Habitats for Captive Chimpanzees," Primates 61 (2020): 139-48, doi:10.1007/s10329-020-00806-5. 
31. Tetsuro Matsuzawa, Masaki Tomonaga, and Masayuki Tanaka, Cognitive Development in Chimpanzees (Tokyo: Springer, 2006), doi:10.1007/4-431-30248-4; Masaki Tomonaga et al., "Development of Social Cognition in Infant Chimpanzees (Pan troglodytes): Face Recognition, Smiling, Gaze, and the Lack of Triadic Interactions," Japanese Psychological Research 46, no. 3 (2004): 227-35, doi:10.1111/ j.1468-5584.2004.00254.x; Satoshi Hirata and Maura Celli, "Role of Mothers in the Acquisition of Tool-Use Behaviours by Captive Infant Chimpanzees," Animal Cognition 6 (2003): 235-44, doi:10.1007/s10071-003-0187-6; and Misato Hayashi and Tetsuro Matsuzawa, "Cognitive Development in Object Manipulation by Infant Chimpanzees," Animal Cognition 6 (2003): 225-33, doi:10.1007/s10071-003-0185-8.

32. Dora Biro and Tetsuro Matsuzawa, "Numerical Ordering in a Chimpanzee (Pan troglodytes): Planning, Executing, and Monitoring," Journal of Comparative Psychology 113, no. 2 (1999): 178-85, doi:10.1037/0735-7036.113.2.178.

33. George Miller, "The Magical Number Seven, Plus or Minus Two: Some Limits on Our Capacity for Processing Information," Psychological Review 63 (1956): 81-97, doi:10.1037/ h0043158.

34. Nobuyuki Kawai and Tetsuro Matsuzawa, "Numerical Memory Span in a Chimpanzee," Nature 403 (2000): 39-40, doi:10.1038/47405.

35. Sana Inoue and Tetsuro Matsuzawa, "Acquisition and Memory of Sequence Order in Young and Adult Chimpanzees (Pan troglodytes)," Animal Cognition 12 (2009): 59-69, doi:10.1007/s10071-009-0274-4.

36. The masking task using Arabic numerals can be a good index of working memory regarding number of numerals and time for remembering. Verbal instruction is minimal: "Please touch the numerals in ascending order." Arabic numerals are universal, which means that we can test memory capacity in terms of developmental changes, aging, and recovery from disease.

37. Sana Inoue and Tetsuro Matsuzawa, "Working Memory of Numerals in Chimpanzees," Current Biology 17, no. 23 (2007): R1004-05, doi:10.1016/j.cub.2007.10.027.

38. Footage of the performance can be viewed online: "Working Memory of Numerals in Chimpanzees," YouTube video, January 21, 2019.

39. There have been numerous ape-language projects in chimpanzees: chimpanzee Gua by Winthrop Kellogg, "Communication and Language in the Home-Raised Chimpanzee," Science 162, no. 3,852 (1968): 423-27, doi:10.1126/ science.162.3852.423; chimpanzee Washoe by Allen Gardner and Beatrice Gardner, "Teaching Sign Language to a Chimpanzee: A Standardized System of Gestures Provides a Means of Two-Way Communication with a Chimpanzee," Science 165, no. 3,894 (1969): 664-72, doi:10.1126/science.165.3894.664; the Washoe project continued by Roger Fouts, "Acquisition and Testing of Gestural Signs in Four Young Chimpanzees," Science 180, no. 4,089 (1973): 97880, doi:10.1126/science.180.4089.978; chimpanzee Sarah by David Premack, Intelligence in Ape and Man (Hillsdale: Lawrence Erlbaum Associates, 1976); chimpanzee Lana by Duane Rumbaugh, Language Learning by a Chimpanzee: The LANA Project (New York: Academic Press, 2014); and chimpanzee Nim by Herbert Terrace et al., "Can An Ape Create a Sentence?" Science 206, no. 4,421 (1979): 891-902, doi: 10.1126/science.504995, and Sue Savage-Rumbaugh, Ape Language: From Response to Symbol (New York: Columbia University Press, 1986). Ape language studies have revealed their cognitive ability to understand other's mind; see David Premack and Guy Woodruff, "Does the Chimpanzee Have a Theory of Mind?" Behavioral and Brain Sciences 1, no. 4 (1978): 515-26, doi:10.1017/S0140525X00076512. Studies reveal physical-causal understanding; see Daniel Povinelli, Folk Physics for Apes: The Chimpanzee's Theory of How the World Works (Oxford: Oxford University Press, 2000). On apes' gestural communication in their natural habitats, see Catherine Hobaiter and Richard Byrne, "The Meanings of Chimpanzee Gestures," Current Biology 24, no. 14 (2014): 1,596-600, doi:10.1016/j.cub.2014. 05.066 .

40. Fumihiro Kano and Masaki Tomonaga, "Face Scanning in Chimpanzees and Humans: Continuity and Discontinuity," Animal Behaviour 79, no. 1(2010): 227-35, doi:10.1016/j.anbehav.2009.11.003; and Fumihiro Kano and Masaki Tomonaga, "Species Difference in the Timing of Gaze Movement Between Chimpanzees and Humans," Animal Cognition 14 (2011): 879-92, doi:10.1007/s10071-011-0422-5.

41. Tetsuro Matsuzawa, "Symbolic Representation of Number in Chimpanzees," Current Opinion in Neurobiology 19, no 1 (2009): 92-98, doi:10.1016/j.conb.2009.04.007.

42. See "The Cognitive Tradeoff Hypothesis," YouTube video, December 5, 2018.

43. Tim White et al., "Ardipithecus ramidus and the Paleobiology of Early Hominids," Science 326, no. 5,949 (2009): 64-86, doi:10.1126/science.1175802.

44. Melissa Emery Thompson et al., "Aging and Fertility Patterns in Wild Chimpanzees Provide Insights into the Evolution of Menopause," Current Biology 17, no. 24 (2007): 2,150-56, doi:10.1016/j.cub.2007.11.033; Kristin Havercamp et al., "Longevity and Mortality of Captive Chimpanzees in Japan from 1921 to 2018," Primates 60 (2019): 525-35, doi:10.1007/s10329-019-00755-8. Long postmenopausal lifespans distinguish humans from all other primates. This pattern may have evolved with mother-child food sharing, a practice that allowed aging females to enhance the fertility of their daughters, thereby increasing selection against senescence. This is the grandmother hypothesis raised by Kristen Hawkes et al., "Grandmothering, Menopause, and the Evolution of Human Life Histories," Proceedings of the National Academy of Sciences 95, no. 3 (1998): 1,336-69, doi:10.1073/pnas.95.3.1336.

45. Aya Saito et al., "The Origin of Representational Drawing: A Comparison of Human Children and Chimpanzees," Child Development 85, no. 6 (2014): 2,232-46, doi:10.1111/ 
cdev.12319; and Tetsuro Matsuzawa, "Pretense in Chimpanzees," Primates 61 (2020): 543-55, doi:10.1007/s10329 -020-00836-z.

DOI: 10.37282/991819.21.51

Published on October 11, 2021

https://inference-review.com/article/primate-memory 